Document downloaded from:

http://hdl.handle.net/10251/179830

This paper must be cited as:

Torres Bosch, MV.; Serral, E.; Valderas, P.; Pelechano Ferragud, V.; Grefen, P. (2020). Modeling of loT devices in Business Processes: A Systematic Mapping Study. IEEE. 221230. https://doi.org/10.1109/CBI49978.2020.00031

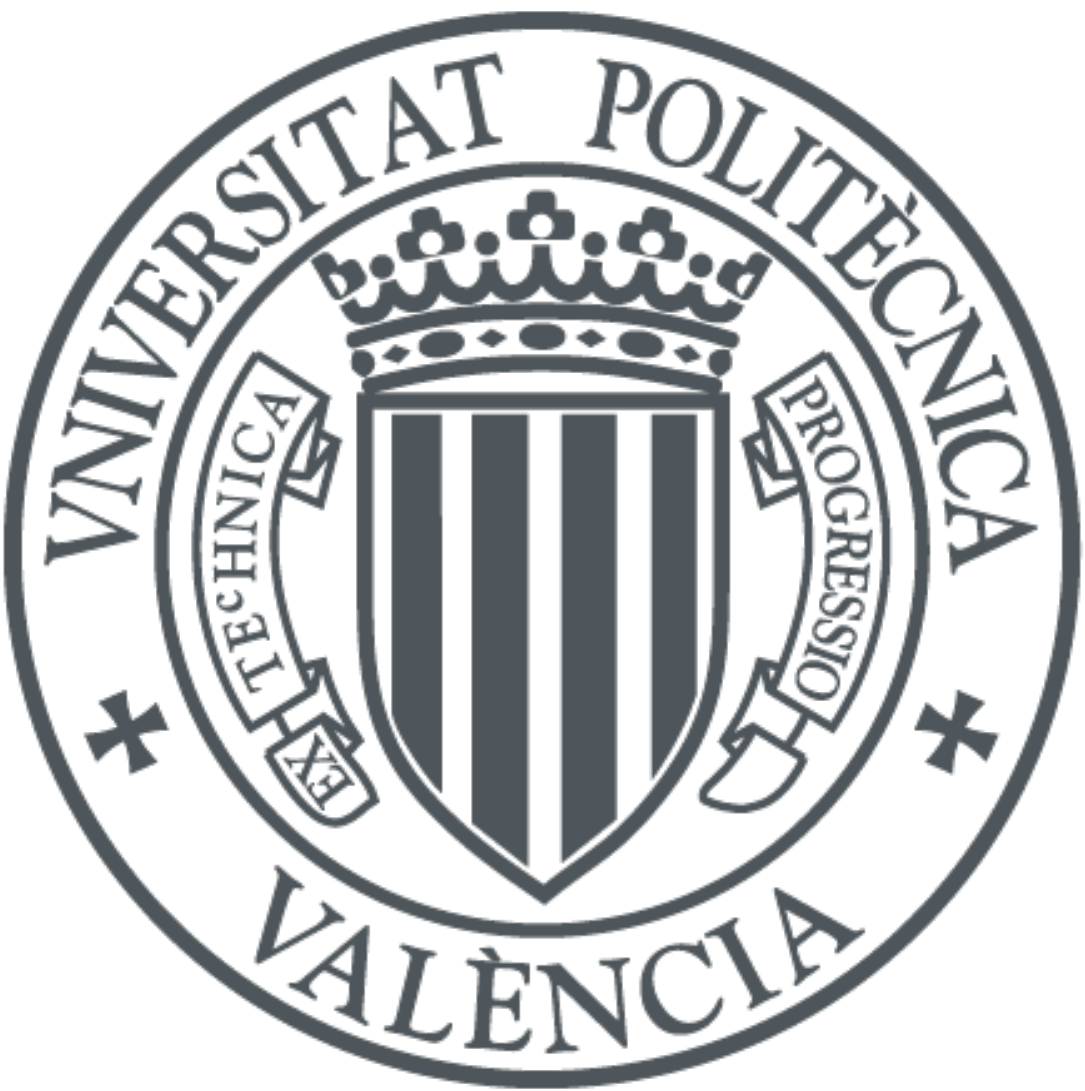

The final publication is available at

https://doi.org/10.1109/CBI49978.2020.00031

Copyright IEEE

Additional Information 


\section{Modeling of IoT devices in Business Processes: A Systematic Mapping Study}

\author{
Victoria Torres \\ PROS Research Centre \\ Universitat Politècnica de València \\ Valencia, Spain \\ vtorres@pros.upv.es
}

\author{
Estefanía Serral \\ LIRIS \\ KU Leuven \\ Leuven, Belgium \\ estefania.serralasensio@kuleuven.be
}

\author{
Pedro Valderas \\ PROS Research Centre \\ Universitat Politècnica de València \\ Valencia, Spain \\ pvalderas@pros.upv.es
}

\author{
Vicente Pelechano \\ PROS Research Centre \\ Universitat Politècnica de València \\ Valencia, Spain \\ pele@pros.upv.es
}

\author{
Paul Grefen \\ School of Industrial Engineering \\ Eindhoven University of Technology \\ Eindhoven, netherlands \\ p.w.p.j.grefen@tue.nl
}

\begin{abstract}
The Internet of Things (IoT) enables to connect the physical world to digital business processes (BP). By using the IoT, a BP can, e.g.: 1) take into account real-world data to take more informed business decisions, and 2) automate and/or improve BP tasks. To achieve these benefits, the integration of IoT and BPs needs to be successful. The first step to this end is to support the modeling of IoT-enhanced BPs. Although numerous researchers have studied this subject, it is unclear what is the current state of the art in terms of current modeling solutions and gaps. In this work, we carry out a Systematic Mapping Study (SMS) to find out how current solutions are modelling IoT into business processes. After studying 600 papers, we identified and analyzed in depth a total of $\mathbf{3 6}$ different solutions. In addition, we report on some important issues that should be addressed in the near future, such as, for instance the lack of standardization.
\end{abstract}

Index Terms-Business process modeling; Internet of Things; IoT devices; IoT-enhanced BP; Systematic mapping study

\section{INTRODUCTION}

Kevin Ashton, who first coined in 1999 the Internet of Things (IoT), envisioned a future where computers could "see, hear, and smell the world for themselves" [1]. Such computer empowerment would not just release human beings from capturing data from the real world but also release computers from their historical dependency on human beings and their limitations to acquire such data. Twenty years later, it is common to find devices and/or things supporting our daily activities both at the personal and professional side. In fact, the IoT device's connection capabilities and their ability to transmit data are revolutionizing the way we live and do business. Even though some of these IoT devices are smart ones capable to react individually upon some events, it is their combined usage what provides an added and innovative value to their users. Within this context, Business Process Management (BPM) appears as an essential component in leveraging the coordination and interaction of IoT devices, so that these can become active participants of future business processes (BPs). According to [2] a BP is defined as "a set of activities that are performed in coordination in an organizational and technical environment. These activities jointly realize a business goal. Each business process is enacted by a single organization, but it may interact with business processes performed by other organizations".

By embracing IoT devices a BP will be able to, e.g., 1) take into account real-world data to take more informed decisions, and 2) automate BP tasks and improve their execution( [3], [4]). In this work we will refer to this type of BPs as IoTenhanced BPs and we define it as a BP that makes use of IoT technology to carry out the process tasks to achieve a specific goal. In the literature this term is referred also as IoT-aware $B P$ (e.g., [5], [6], [7], [8], [9], [10], [11], [12], [13]). However, in this work we prefer to use the term IoT-enhanced BP since we understand that BPs in this context are more than informed or alerted by existing IoT elements but magnified by the use of these elements, increasing as a result their value and quality.

As a first approach to merge the IoT and the BPM fields, in this work we focus on the design and analysis phase from the BPM lifecycle [2], specifically on the modeling task. We expect that the specificity of the topic being addressed will restrict the potential candidates studies for the analysis, allowing for an in-depth study of the selected proposals. As a result, we carry out a Systematic Mapping Study (SMS) where we carefully review the state of the art developed in this topic. Therefore, the major objective of this work is having an indepth understanding of the current and common trends that exist to properly modeling IoT devices into BPs i.e. to clearly represent such new players within a BP model..

\section{A. Research questions}

In the literature we can find many definitions of the IoT term (e.g., [14], [15], [16], [17]). However, in a broad sense, the IoT is characterized by a network of interconnected computing devices that are seamlessly integrated in so-called things, any 
real-world item (i.e., physical object, animal, person, etc.) which can be attached a computing device to. Depending on its capabilities towards the associated real-world item, a computing device can be categorized as a tag, to identify the associated physical entity (e.g., product id), as a sensor, to provide data about the associated physical entity that is being monitored (e.g., temperature, ambient noise level, location, lighting, hearth rate, blood pressure, etc.), or as an actuator, to provide mechanisms that allow modifying the state of the associated physical entity (e.g., increasing/reducing the heat of a room, switching on/off room lighting, switching on/off garden irrigation, etc.).

Taking into account the important role that the IoT device concept play in the IoT domain, it needs to be somehow present in IoT-enhanced BP models. To overcome the challenge of introducing such concept at the modeling level, we also need to understand the used modeling strategies. Thus, we state the following two research questions:

RQ1. Which modeling strategies are provided to build IoT-enhanced BPs?

The generic-purpose constructs provided by common BP modeling languages such as Petri Nets, EPC, Yawl, BPEL, BPMN, UML Activity Diagram (UMLAD) may not be suitable to represent IoT-enhanced BPs. There are different ways to overcome this limitation, e.g., by extending existing BP languages with new constructs, by creating a new domain specific language (DSL), or by complementing BP models with other models to represent such specific aspects separately from the BP specification. Therefore, the objective of RQ1 is to identify the strategies used to design a modeling solution for the construction of IoT-enhanced BP models and also which BP languages are most preferred for implementing the chosen strategy.

RQ2. How IoT devices are represented at the modeling level?

Within the IoT domain we find a plethora of things and devices that play an active role for the achievement of a specific goal, for example by taking the responsibility of some of the tasks that make up a specific BP. Therefore, the objective of RQ2 is identifying how IoT devices can be represented when modeling IoT-enhanced BPs.

\section{B. Structure of the paper}

The remainder of the paper is organized as follows. Section II describes the research methodology applied to conduct the SMS. Section III answers the RQs by analyzing the set of primary studies resulting from the literature search. Section IV discusses the results from the SMS. Section V summarizes the state of the art, stressing the differences with our SMS. Finally, section VI presents our conclusions.

\section{Methodology}

To conduct the SMS we have followed the guidelines, procedures, and policies proposed by Kitchenham in [18]. Accordingly, we have defined a review protocol that starts with the specification of the research questions (cf. Section I-A). Then, a search string was defined to identify, as much as possible, all relevant literature (cf. Section II-A) from a selection of data sources (cf. Section II-B). As a result we obtained a list of studies that was evaluated by the authors, based on title and abstract, for inclusion in the set of candidate papers. Inclusion and exclusion criteria (cf. Section II-C) have been defined to assess each potential primary study. As a result of this stage, an initial set of potential primary studies was obtained. In addition, to reduce the probability of missing relevant studies, we have complemented the initial search with backward and forward snowballing [19].

\section{A. Search string}

The search string defined in this study has been elaborated based on keywords we derived from our own knowledge on the topic, i.e., we applied subjective search string definition ( [20]). In addition, to broadly cover the scope of the SMS we have used a wide range of synonymous terms which have been connected through the OR logical connector. The final search string has been obtained after refining it iteratively with the goal of maximizing the number of relevant studies to be analysed in this SMS. This refinement has been performed with pilot searches with the search string based on a trial and error approach, excluding all those terms that do not contribute to retrieve any additional studies.

As a result, the search string applied during the SMS was the following:

\section{((bpmn OR "uml activity” OR epc OR yawl OR "petri nets” OR bpel) OR (process OR workflow OR "service composition")) AND modeling AND (iot OR "internet of things" OR "cyber physical system” OR ubiquitous OR pervasive OR "smart system" $O R$ "ambient intelligence" $O R$ "context adaptive" OR "context aware")}

To increase the probability of retrieved studies dealing with these terms, we applied the proposed search string to two meta-data fields, i.e. keywords and title. The automated search was performed with Papers $^{1}$, a reference management software that allows automating searches across multiple search engines.

\section{B. Data source selection}

The relevant studies for this SMS are those published in proceedings of the most relevant conferences, and workshops, and journals where the BPM and the IoT communities are present. These include, among others, 1) journals such as Data \& Knowledge Engineering, Computers in Industry, Personal and Ubiquitous Computing, Service Oriented Computing and Applications, Information Systems, Information and Software

\footnotetext{
${ }^{1}$ https://www.readcube.com/papers/
} 
Technology, and 2) conferences and workshops such as the International Conference on Business Process Management (BPM), the International Conference on Service-Oriented Computing (ICSOC), the Advances in Grid and Pervasive Computing Conference (GPC), the Ubiquitous Computing and Ambient Intelligence Conference (UCAmI), the Embedded and Ubiquitous Computing Conference (EUC), Conference on Advanced Information Systems Engineering Conference(CAiSE), the Working Conference of Business Process Modeling, Development, and Support (BPMDS), the IEEE Enterprise Computer Conference (EDOC), the International Conference on Cooperative Information Systems (CoopIS), the Symposium on Applied Computing (SAC), the International Conference on Service Computing (SCC), the International Workshop on BP-Meet-IoT (BP-meet-IoT), the International Workshop on Business Process Modeling Notation (BPMN), and the IEEE International Workshops on Enabling Technologies: Infrastructure for Collaborative Enterprises (WETICE). All these journals, conferences, and workshops are registered in the following five electronic libraries, which have been used to conduct this SMS: SpringerLink, ScienceDirect, Scopus, Google Scholar, and Crossref Search.

With this selection of libraries we want to ensure a broad coverage, so we could retrieve the maximum number of candidate studies from a minimum number of libraries. As an additional data source, we considered the literature referenced by the retrieved studies and the literature that cited the retrieved studies; i.e., literature found by the application of backward and forward snowballing.

\section{Inclusion and exclusion criteria}

We defined several inclusion and exclusion criteria in order to follow a systematic approach to select the studies that must be considered as relevant. Therefore, all the studies meeting any of the exclusion criteria are eliminated from the analysis process. In particular, the following criteria were considered:

\section{1) Inclusion criteria:}

The study describes a modeling approach dealing with IoT-enhanced BPs.

2) Exclusion criteria:

a) The study is not focused on the modeling of IoT-enhanced BPs or merely mentioning modeling in a general manner.

b) The study does not include sufficient data to infer how IoT is taken into account in the BP model.

c) In case of several studies referring to the same modeling approach, all studies, except the latest and most complete version, are excluded.

d) The study is published in a non-peer reviewed publication (e.g., a preface, editorial, or technical report).

e) The study is not written in English.

f) The study is presented as a short paper of less than 5 pages.

g) The study is not electronically available or requires payment of access fees ${ }^{2}$.

\footnotetext{
${ }^{2}$ Note that this only applies to fees that are not covered by the subscriptions to any of the selected data sources.
}

\section{Study selection}

We applied the proposed methodology through 5 stages. Data extraction was performed by the two first authors who collected such data in an Excel spread sheet. This sheet was reviewed by the remaining authors to check the decisions made over each study After stage 1, a total number of 600 studies were retrieved from the five electronic libraries selected. Then, in stage 2, after applying the items d-g identified in the exclusion criteria, a total of 534 studies were discarded, obtaining as a result a total of 66 potential studies. Afterwards, in stage 3 , these potential studies were evaluated based on their title, abstract, and keywords in order to determine its relevance to the SMS according to the inclusion criteria and items a-c from the exclusion criteria. As a result, a set of 20 studies were selected. Then, from this selection we performed backward and forward snowballing to analyze the referenced literature (stage 4) and the literature that cited the primary studies (stage 5). This last stages resulted in the incorporation of 16 new studies, resulting in a total of 36 studies which are summarized in Table I. In addition to the reference to each study, this table shows, the name of the corresponding proposal, the publication year (Yr), the type of venue where the proposal has been published (i.e., journal $(\mathrm{Jr}$.), conference $(\mathrm{Cn}$.$) , and workshop (\mathrm{Wr}$.$) ) and finally the$ major research field to which the publication venue belongs to. In this case we have categorized the venues into three groups, i.e., IoT for venues where research is focused around IoT technology, BPM for venues where the topics are built around BPs and services, and IT for venues with a broader scope covering usually both fields.

\section{RESULTS}

According to the two RQs proposed in section I-A, here we present the major results obtained after analyzing the 36 primary studies selected for this SMS. As Table II shows, there is a clear increase of proposed approaches in the last five years $(72,2 \%$ of the works have been published between 2015 and 2019). In addition, the type of venues selected to publish these studies include workshops, conferences, and journals. In this case, most of the studies $(80,6 \%)$ have been published in conferences $(41,7 \%)$ and journals $(38,9 \%)$, revealing the maturity of the published works. Finally, the research areas of the selected venues include the BPM area (e.g., Information Systems journal (IS), BPM conference, BPMN workshop or CAiSE conference), the IoT area (e.g., ICNSC conference, DASC conference, ICNSC conference or the journal IEEE Transactions on industrial informatics), and also the IT area where more generic venues are considered such as the Journal of Universal Computer Science. In this case, as we may expect, most of the studies $(72,3 \%)$ have been published in venues belonging to the more specific areas, i.e., BPM $(41,7 \%)$ and IoT $(30,6 \%)$. 


\begin{tabular}{|c|c|c|c|c|}
\hline \multirow{2}{*}{ Study ID } & \multirow{2}{*}{ Proposal's name } & \multicolumn{3}{|c|}{ Publication } \\
\hline & & $\mathbf{Y r}$ & Type & Field \\
\hline Al-alshuhai2015 [21] & Context-aware AD & '15 & $\mathrm{Cn}$. & IoT \\
\hline Albreshne2015 [22] & GPL4SRE & '15 & Cn. & IoT \\
\hline Appel2014 [23] & SPUs & '14 & Jr. & BPM \\
\hline Baresi2015 [24] & - & 15 & Wr. & BPM \\
\hline Bocciarelli2017 [25] & PyBPMN & '17 & Cn. & IoT \\
\hline Breitenbücher2015 [26] & SitME4BPEL & '15 & Cn. & IT \\
\hline Bucchiarone2009 [27] & APFoL & '09 & Wr. & BPM \\
\hline Caracaş2011 [28] & - & '11 & Wr. & BPM \\
\hline Cheng2019 [11] & - & 19 & Jr. & IoT \\
\hline Chiu2015 [9] & - & 15 & Cn. & IoT \\
\hline Dar2015 [29] & - & 15 & Jr. & IoT \\
\hline Domingos2014 [30] & - & 14 & Jr. & IT \\
\hline Domingos2017 [12] & - & '17 & Jr. & IT \\
\hline Dörndorfer2018 [31] & Context4BPMN & '18 & Conf. & BPM \\
\hline Friedow2018 [32] & - & '18 & $\mathrm{Cn}$. & BPM \\
\hline Gao2011 [33] & - & '11 & Wr. & BPM \\
\hline Graja2016 [34] & BPMN4CPS & 16 & $\mathrm{Cn}$. & BPM \\
\hline Kefalakis2011 [35] & APDL & '11 & Jr. & IT \\
\hline Kim2014 [36] & - & 14 & Cn. & IoT \\
\hline Kim2016 [37] & Process-aware IoT & '16 & Jr. & IT \\
\hline Lee2016 [38] & BPMN-MDM & 16 & Jr. & IoT \\
\hline Maamar2018 [39] & PoT & '18 & Cn. & BPM \\
\hline Mandal2017 [40] & - & 17 & Cn. & BPM \\
\hline Meyer2013 [41] & - & 13 & Cn. & IT \\
\hline Mottola2018 [42] & makeSense & '18 & Jr. & IT \\
\hline Petrasch2016 [43] & I4PML & 16 & Cn. & BPM \\
\hline Sasirekha2016 [44] & - & 16 & $\mathrm{Cn}$. & IoT \\
\hline Schönig2018 [7] & - & '18 & Cn. & BPM \\
\hline Seiger2015 [45] & - & 15 & Jr. & IT \\
\hline Serral2015 [46] & CAPN & '15 & Jr. & IoT \\
\hline Sperner2011 [47] & - & '11 & Wr. & BPM \\
\hline Suri2017 [48] & IoT-BPO & '17 & $\mathrm{Cn}$. & BPM \\
\hline Tu2018 [6] & IoTPM & 18 & Jr. & IT \\
\hline Wang2014 [49] & CWfMS & 14 & Jr. & IoT \\
\hline Wehlitz2017 [5] & - & '17 & Wr. & BPM \\
\hline Yousfi2019 [50] & UDABP & 19 & Jr. & IT \\
\hline
\end{tabular}

SMS PRIMARY STUDIES

\begin{tabular}{c|c|c||c|c|c||c|c|c|}
\cline { 2 - 9 } & \multicolumn{2}{c||}{ Year } & \multicolumn{3}{c||}{ Venue type } & \multicolumn{3}{c|}{ Venue field } \\
\cline { 2 - 9 } & $\mathbf{0 9 - 1 4}$ & $\mathbf{1 5 - 1 9}$ & Cn & Wr & Jn & BPM & IoT & IT \\
\hline \hline$\#$ & 10 & 26 & 15 & 7 & 14 & 15 & 11 & 10 \\
\hline$\%$ & 27,8 & 72,2 & 41,7 & 19,4 & 38,9 & 41,7 & 30,6 & 27,8 \\
\hline \hline
\end{tabular}

SUMMARY OF PUBLICATION YEAR, VENUE TYPE AND VENUE AREA

\section{A. Modeling strategies used (RQ1)}

In this section we focus on the modeling strategies that are used by the primary studies to model IoT-enhanced BPs. Based on the analysis we have categorized the studies into two main groups, one related to the studies that build their modeling proposal from the most well-known general purpose BP modeling languages, henceforth Built-from-existing, and a second one that relates to studies that build a new domain specific language (DSL), henceforth Build-dsl. While most of the studies $(80,56 \%)$ fall within the Built-from-existing category (29 out of 36), just a small amount $(19,44 \%)$ is classified within the Build-dsl category (7 out of 36). Tables III and IV gather the studies that fall into the Built-from-existing and Build-dsl categories respectively.

Regarding the Built-from-existing category, the type of use

\begin{tabular}{l|c|c|c|c|c|}
\hline Study ID & BPMN & EPC & BPEL & UMLAD & PN \\
\hline \hline Al-alshuhai2015 & & & & $\nearrow$ & \\
\hline Appel2014 & $\nearrow$ & $\nearrow$ & & & \\
\hline Baresi2015 & $\equiv$ & & & & \\
\hline Bocciarelli2017 & $\nearrow$ & & & & \\
\hline Breitenbücher2015 & & & $\nearrow$ & & \\
\hline Bucchiarone2009 & & & $\nearrow$ & & \\
\hline Caracaş2011 & $\equiv$ & & & & \\
\hline Cheng2019 & $\nearrow$ & & & & \\
\hline Chiu2015 & $\nearrow$ & & & & \\
\hline Dar2015 & $\equiv$ & & & & \\
\hline Domingos2014 & & & $\nearrow$ & & \\
\hline Domingos2017 & $\equiv$ & & & & \\
\hline Dörndorfer2018 & $\nearrow$ & & & & \\
\hline Friedow2018 & $\equiv$ & & & & \\
\hline Gao2011 & $\oplus$ & & & & \\
\hline Graja2016 & $\nearrow$ & & & & \\
\hline Kim2014 & & & $\nearrow$ & & \\
\hline Lee2016 & $\nearrow$ & & & & \\
\hline Manda12017 & $\nearrow$ & & & & \\
\hline Meyer2013 & $\nearrow$ & & & & \\
\hline Mottola2018 & $\nearrow$ & & & & \\
\hline Petrasch2016 & $\nearrow$ & & & & \\
\hline Sasirekha2016 & & & $\equiv$ & & \\
\hline Schönig2018 & $\nearrow$ & & & & \\
\hline Serral2015 & & & & & $\oplus$ \\
\hline Sperner2011 & $\nearrow$ & & & & \\
\hline Suri2017 & $\nearrow$ & & & & \\
\hline Wehlitz2017 & $\oplus$ & & & & \\
\hline Yousfi2019 & $\nearrow$ & & & & \\
\hline \hline Number of studies & 22 & 1 & 5 & 1 & 1 \\
\hline$\%$ of studies & 73,33 & 3,33 & 16,67 & 3,33 & 3,33 \\
\hline
\end{tabular}

Legend: $\operatorname{Extends}(\nearrow)$; As-is $(\equiv)$; References $(\oplus)$ TABLE III

PRIMARY STUDIES BELONGING TO THE Built-from-existing CATEGORY

proposals do over existing BP modeling languages vary. In particular, we have identified three different types of uses which we have catalogued as (1) Extends, to refer to those studies that propose enriching an existing modeling language with new concepts closer to the new requirements imposed by IoT systems, (2) As-is, to refer to the studies that propose using a specific modeling language in its original shape, with no changes or extensions, and (3) References to refer to those works that amplify the BP model by introducing new elements to linking it with another modeling artefact.

Table III classifies those primary studies falling within the Built-from-existing category. Note that Appel2014 extends two BP modeling languages (BPMN and EPC). For this reason we are counting this study twice in the analysis regarding the usage of existing BP modeling languages, having as a result 30 studies instead of 29 . As this table shows $73,33 \%$ of the studies (22 out of 30 ) use BPMN, and only $26,67 \%$ ( 8 out of 30 ) use the remaining languages as follows $16,67 \%$ for BPEL, and 3,33\% for EPC, UML Activity Diagram (AD), and PN respectively.

Regarding the studies that belong to the second category (Build-dsl), Table IV details the metamodeling language used in each case to build the proposed DSL. In many cases these proposals take concepts from existing languages to build their own metamodel such as Kefalakis2011 with XPDL [51], 


\begin{tabular}{l|c|c|c|c|c|c|}
\hline Study ID & n/s & Ecore & OWL & E-R & XML & ABNF \\
\hline \hline Albreshne2015 & $\mathrm{x}$ & & & & & \\
\hline Kefalakis2011 & & & & & $\mathrm{X}$ & \\
\hline Kim2016 & & & & $\mathrm{x}$ & & \\
\hline Maamar2018 & & & & & & $\mathrm{x}$ \\
\hline Seiger2015 & & $\mathrm{x}$ & & & & \\
\hline Tu2018 & $\mathrm{x}$ & & & & & \\
\hline Wang2014 & & & $\mathrm{x}$ & & & \\
\hline
\end{tabular}

PRIMARY STUDIES BELONGING TO THE Build-dsl CATEGORY

Seiger2015 with BPMN and EPC, and Maamar2018 with Storytelling [52].

However, not all the proposals rely just on a single modeling artefact to specify IoT-enhanced BPs. In fact, some primary studies apply the separation of concerns (SoC) design principle to design their modeling proposals. As a result different models targeted at different aspects are combined to properly specify such systems. In particular, the studies propose combining modeling artefacts as follows:

Albreshne2015 proposes combining the GPL4SRE, which is the modeling proposal based on BPEL4WS to describe BPs, with the ontology Ont4SRE to describe "smart objects". Baresi2015 proposes the use of an extended Guard-StageMilestone (GSM) model to model and monitor the BP part that refers to goods that are moving from different organizations. These goods are turned into smart objects since these are equipped with software running, sensing data and communication capabilities. Dörndorfer2018 proposes the sensor model (SenSoMod) to specify sensors, context and how these relate to each other. For example, by means of this model context data can be defined as an aggregation from data retrieved by different sensors. This aggregation is achieved through the context description element which is included in both, the BPMN extension (Context4BPMN) and also in the SenSoMod model and is used as a mechanisms to link both models. Gao2011 proposes linking BPMN models with the Functional Model to import a sensor ontology and its instance data. Sasirekha2016 proposes combining BPEL models with an ontology that defines IoT entities and that is integrated with the SSN ontology. Serral2015 defines CAPN, a proposal that integrates CPN with ontologies to describe context. Suri2017 proposes providing a semantic description of the BPMN models by means of an ontology that integrates concepts from the BP and the IoT domains. This integrated ontology is built from IoTBPO (an ontology defined from the IoT resources (Sensor, actuator y tag) included in the defined BPMN extension), BPMO [53], and IoT-Lite [54] ontologies. Tu2018 combines three models specified at three different layers to represent separately the domain (IoTCM), the process (IoTPM), and objects (IoTOM). Yousfi2019 combines their BPMN extended proposal (uBPMN) with a Decision Model where ubiquitous decisions, i.e., decisions taken based on an important amount of data (e.g., location, traffic status, gas level, etc.) are defined to improve the BP.

\section{B. IoT device representation (RQ2)}

According to our analysis, IoT devices (i.e., smart objects, physical entities, and devices) can be either represented in the BP model explicitly, i.e., by dedicating specific modeling elements to refer to such concepts or implicitly, i.e., by means of modeling elements that behave as bridges between the BP model and the physical entity or device. While $61,11 \%$ of the studies (22 out of 36) fall into the former category, 38,89\% of the studies (14 out of 36) fall into the latter. Based on these two major categories we explain next how the different studies deal with such representation.

1) Explicit representation of IoT devices: During the analysis we identified that IoT devices are represented explicitly by means of the following mechanisms: a) by extending data objects (Do), b) by extending resources or participants which are represented graphically as pools or lanes $(\mathrm{P} / \mathrm{L})$, c) defining new constructs $(\mathrm{Nc})$ in the metamodel, or d) by representing them in a separated model $(\mathrm{Sm})$ that is linked to the BP specification. Table V categorizes the different studies according to these four mechanisms.

We have found 2 studies that extend data objects. Lee2016 proposes to extend the Multipe-Domain Matrix (MDM) with BPMN modeling elements to describe elements and relationships between business processes. The activity DSM element from the matrix has been designed taking as reference the BPMN language and extending the data object element to represent smart objects. Yousfi2019 extends the BPMN data object to represent explicitly smart objects. In addition, the BPMN activity and event elements are also extended to allow specifying IoT input technologies such as sensor, smart readers and so on. With this extension, activities and events can be specialized into one of the following subtypes: Sensor, Reader, Image, Audio, and Collector.

Regarding those that extend the resource or participant, 8 studies were found. Al-alshuhai2015 extends a resource which is represented with swim-lanes. It proposes representing sensing devices by means of swim-lanes that they call context source (CS) segments. These lanes gather activities that represent actions (e.g., connect to the sensor, acquire a sensed measure, etc.) with the device. Chiu2015 extends a resource which is represented with a lane. It allows representing physical entities (e.g., a room) as BPMN resources, represented graphically as swimlanes. In addition, sensing and actuation activities over those resources are represented following the Meyer et al. [10] proposal, i.e. by means of sensing and actuation tasks, which are tasks defined as an extension to the BPMN activity element. Domingos2017 extends a participant which is represented with a pool. It proposes representing IoT devices as BPMN participants, i.e., as processes modeled in separated pools. Within each pool, BPMN script tasks are used to represent the interaction required with the corresponding device (e.g., switching on/off, measuring a particular property, etc.). Kim2014 extends the BPEL partnerLinks element with a new participant, the IoTService to specify different types of devices (e.g., robots, smartphones, bio-mobile, and sensors). 
This new participant defines the deviceType element where the device id, type and model can be specified. Meyer2013 extends a participant which is represented with a pool. This work represents physical entities by extending the BPMN metamodel with the PhysicalEntity concept. This new concept specializes the ParticipantContainer class which is also introduced in the metamodel as a superclass for the BPMN Participant class. The PhysicalEntity concept is represented graphically as an empty collapsed pool that interacts with process flow elements contained in a separated pool called IoT Process. The interaction with such entities is modeled through Sensing Tasks, representing information sent from the Physical Entity to the IoT Process and Actuation Tasks, representing information sent by the IoT Process to the Physical Entity. Petrasch2016 represents IoT devices as BPMN partitions in the BP model. In addition, to represent the interaction with such devices, it proposes to extend the BPMN notation into Sensing tasks and Actuating tasks. These are depicted graphically with a differentiating icon placed on the top-left corner of the task. Suri2017 extends the BPMN Resource element with the ResourceExtension to include IoT Devices (i.e., Sensor, Actuator, and Tag) and also their quality attributes (e.g., accuracy, response time, etc.) into the BP model. This extended element is represented graphically as a new element that is associated to BP tasks. Wehlitz2017 extends a resource which is represented with a line. In particular, this work proposes to use BPMN swimlanes to represent device types which are represented at both, the type and instance level. While the type level description is used to represent similar devices of the same type (e.g., a temperature sensor), the instance level allows describing concrete devices in the model (e.g., the temperature sensor installed in the living room). This allows defining instance-independent BP models that can be reused at design time.

Other 7 studies propose new contructors. Albreshne2015 includes in their proposed language (GPL4SRE) a section called Smart Entities Declaration where smart entities (e.g., a lamp, a room, etc.) can be explicitly declared. Bocciarelli2017 extends the PyBPMN language (a BPMN extension to address performance and reliability analysis) to model resources, i.e., real entities that perform activities in the process from the I4.0 perspective. In particular, the PyCPS class is introduced as a BPMN Resource specialization to represent real entities participating in a BP. Then, a PyCPS class is made of a set of components which include sensors and actuators, represented by the PyCPS_Sensor and PyCPS_Actuator metaclasses respectively. Bucchiarone2009 extends their proposed APFoL language to allow specifying by means of context entities those physical entities (e.g., boxes, warehouse, etc.) whose state is of interest to the process (e.g., if the box has been damaged or not). Cheng2019 focuses on sensor networks providing a BPMN extension that includes: the Sensor Device class to determine the type of sensor being represented (e.g., a light sensor or a pressure sensor), the Sensor Service class to define the function provided by the sensor device, and the Handler class, to specify the technology used to access the sensor service (e.g., Restful, URL). Graphically, a sensor task is differentiated from an standard BPMN task with a sensor icon which is placed on the top-left corner of the task. In this case the Sensor Device has been included in the metamodel as an element that aggregates BPMN Activities and also Handlers. Finally, the Sensor Services concept is defined as a class that aggregates the BPMN Performer class. Kim2016 differentiates in their proposed metamodel the Things concept into Virtual Things, i.e., smart objects, and Physical Thing, which can be refined into a person, sensor, actuator, device, service or other type of thing. Maamar2018 defines things (either living or non-living) through the Character and SelectedCharacter elements included in the PoT's scripts (at design-time) and scenes (at run-time) respectively. In this case all things relevant for the scene are included either through an automatic or manual detection. Sperner2011 proposes to extend the BPMN metamodel to represent physical entities and their interaction with devices (i.e., sensors and actuators). On the one hand physical entities are represented by the PhysicalObject new concept, which can be used to represent single objects but also a collection of objects. Graphically this new concept is represented as a rectangular box which includes three vertical bars at the bottom of its front face to denote collections. On the other hand, the BPMN Task element has been extended as SensingTask, to provide the process with data monitored from a physical entity, and ActuatingTask, to act upon a physical entity.

Finally, 5 studies propose a separate model to represent IoT devices. Dörndorfer2018 proposes a separate model to specify sensors and its relation with context through the Sensor Model. This separated model is linked to BP model specified in Context4BPMN, an extension to BPMN to allow the creation of context-aware BPs. The type of sensors that can be specified in the Sensor Model include atomic (physical and virtual) and computed, obtained from the aggregation of multiple sensors. Gao2011 proposes a separate model to enrich BPMN models with sensor and smart devices and their provided data by means of the Business Functional Model (BFM). The BFM define attribute-featured entities via a set of properties and their values, which are related in some cases to properties of the SSN ontology. Sasirekha2016 represents IoT devices by means of an ontology. Such ontology is defined as the integration of a defined domain ontology and the SSN ontology. Serral2015 represents devices in an ontology. Petri nets are used to model business processes and they are extended to include conditions over the context sensed by the specified devices. Tu2018 proposes to build the so called IoT-aware Ontology Concept Model (IoTCM) to representing semantically the domain, in particular the involved Objects, Resources, and Business Entities. Then, the IoT-aware Process Model (IoTPM) is built by referencing those elements in the corresponding IoT Process Steps included in the model.

2) Implicit representation of IoT devices: Within this category we find the studies that represent IoT devices implicitly through their interaction. Accordingly, two different mechanisms are identified to achieve such interaction, i.e., through 
tasks and through events. Table $\mathrm{V}$ categorizes the different studies according to these two mechanisms.

There are 6 studies that are based on the task concept. Breitenbücher2015 proposes, in the SitME modeling language, to attach real entities to situation events and situational scopes, two new types of events that get triggered when a specific situation occurs for the associated entity. However, when SitME is translated to BPEL, these new types of events are transformed into BPEL receive and invoke activities respectively. Dar2015 proposes integrating smart objects into BPs through the use of BPMN script tasks. These tasks are in charge of collecting measured data from IoT devices and transmit it where need it. These tasks can be contained either in the the Set Top Box (STB) BP model part and in the smart phone BP model part, which are connected through messages to pass the measurement responsibility depending on the user location. Domingos2014 introduces the concept of IoT aware processes where actions with devices are modelled through the invocation of web services. Graja2016 represents the interaction with physical devices by extending BPMN service tasks. In particular, the extension includes physical tasks, tasks that are refined into actuator and sensor tasks, and cyber tasks, tasks that are executed by a piece of software, i.e., a web service, cloud service or embedded service. Mottola2018 extends BPMN with the WSN task concept to represent interactions with the WSN. These tasks are differentiated graphically from standard BPMN tasks by including an antenna icon in their top-left corner. All these tasks are contained within a new type of pool, the WSN Pool, introduced in the notation to separate the business logic that is performed by the WSN from the logic performed by traditional IT systems. This new pool includes the same antenna icon to graphically differentiate it from an standard BPMN pool. Seiger2015 provides a solution based on tasks. It includes in its DSL the Atomic Process Step concept which is used to integrate services and devices into Processes. This concept specializes the Process Step concept which is the basic component for modeling processes.

Other 8 studies are based on the concept of Event. Schönig2018 proposes an architecture where BPs get notified after subscription on certain object's state changes. Appel2014 introduces the Event Stream Processing Units (SPUs) abstract concept to integrate relevant environmental data into the process. SPUs encapsulate event stream processing in BP models. Baresi2015 proposes to use the infrastructure deployed in the own smart objects to sense the environment and trigger events. This infrastructure includes the trace generator component which consists of a CEP engine that compares data streams with sentries (conditions expressed as Boolean formulas) defined in the process model to detect process events. The conditions that define when an event is triggered is specified in the guards and milestones defined in the extended GSM model. Caracaş2011 makes use of the different types of events provided in BPMN (i.e., escalation, timer, and message events) to capture the reactive nature of the WSN applications. Friedow 2018 proposes to use the Bosch IoT Things service to define the connection between IoT

\begin{tabular}{|c|c|c|c|c|c|c|}
\hline \multirow{2}{*}{ Study ID } & \multicolumn{4}{|c|}{ Explicit } & \multicolumn{2}{|c|}{ Implicit } \\
\hline & Do & $\mathbf{P} / \mathbf{L}$ & Nc & Sm & Tk & Ev \\
\hline Al-alshuhai2015 & & $\mathrm{x}$ & & & & \\
\hline Albreshne2015 & & & $\mathrm{x}$ & & & \\
\hline Appel2014 & & & & & & $\mathrm{x}$ \\
\hline Baresi2015 & & & & & & $\mathrm{x}$ \\
\hline Bocciarelli2017 & & & $\mathrm{x}$ & & & \\
\hline Breitenbücher2015 & & & & & $\mathrm{x}$ & \\
\hline Bucchiarone2009 & & & $\mathrm{x}$ & & & \\
\hline Caracaş2011 & & & & & & $\mathrm{x}$ \\
\hline Cheng2019 & & & $\mathrm{x}$ & & & \\
\hline Chiu2015 & & $\mathrm{x}$ & & & & \\
\hline Dar2015 & & & & & $\mathrm{x}$ & \\
\hline Domingos2014 & & & & & $\mathrm{x}$ & \\
\hline Domingos2017 & & $\mathrm{x}$ & & & & \\
\hline Dörndorfer2018 & & & & $\mathrm{x}$ & & \\
\hline Friedow2018 & & & & & & $\mathrm{x}$ \\
\hline Gao2011 & & & & $\mathrm{x}$ & & \\
\hline Graja2016 & & & & & $\mathrm{x}$ & \\
\hline Kefalakis2011 & & & & & & $\mathrm{x}$ \\
\hline Kim2014 & & $\mathrm{x}$ & & & & \\
\hline Kim2016 & & & $\mathrm{x}$ & & & \\
\hline Lee2016 & $\mathrm{x}$ & & & & & \\
\hline Maamar2018 & & & $\mathrm{x}$ & & & \\
\hline Mandal2017 & & & & & & $\mathrm{x}$ \\
\hline Meyer2013 & & $\mathrm{x}$ & & & & \\
\hline Mottola2018 & & & & & $\mathrm{x}$ & \\
\hline Petrasch2016 & & $\mathrm{x}$ & & & & \\
\hline Sasirekha2016 & & & & $\mathrm{x}$ & & \\
\hline Schönig2018 & & & & & & $\mathrm{x}$ \\
\hline Seiger2015 & & & & & $\mathrm{x}$ & \\
\hline Serral2015 & & & & $\mathrm{x}$ & & \\
\hline Sperner2011 & & & $\mathrm{x}$ & & & \\
\hline Suri2017 & & $\mathrm{x}$ & & & & \\
\hline Tu2018 & & & & $\mathrm{x}$ & & \\
\hline Wang2014 & & & & & & $\mathrm{x}$ \\
\hline Wehlitz2017 & & $\mathrm{x}$ & & & & \\
\hline Yousfi2019 & $\mathrm{x}$ & & & & & \\
\hline Number of studies & 2 & 8 & 7 & 5 & 6 & 8 \\
\hline$\%$ of studies & 5,6 & 22,2 & 19,4 & 13,9 & 16,7 & 22,2 \\
\hline Number of studies & & & 2 & & & \\
\hline$\%$ of studies & & & & & & \\
\hline
\end{tabular}

Legend:

Do: Data object; P/L: Pool/Lane;

Nc: New construct; Sm: Separated model;

Tk: Task; Ev: Event;

TABLE V

IOT DEVICE REPRESENTATION SUPPORT PROVIDED BY PRIMARY STUDIES

devices and the BP by influencing the execution of BPs by the reception of events. Kefalakis2011 associates different types of events that refer to physical objects to process activities which are called Elementary Business Processes (EBProc) in APDL. Mandal2017 proposes a framework based on the processing of events, which are captured by BP tasks that are subscribed to it. Wang2014 includes in their proposed architecture the context provisioning platform $(C P P)$ which behaves as intermediary between sensors and BPs through the triggering of events that include high-level context data.

Table V summarizes the number of studies and percentages that fall into the two major categories, detailing also the modeling element or mechanism used to represent IoT devices. 


\section{DISCUSSION}

Regarding RQ1, we have learned that BPMN is by far the modeling language preferred by most of the analyzed studies $(73,33 \%)$. Other BP languages used to a lesser extent are BPEL, EPC, Petri Nets, and UMLAD. This is not a surprise if we consider that BPMN is widely used standard that provides a graphical notation and extension capabilities, a key aspect for this research question. In fact, this capability has been used by most of the solutions to give support to the newly introduced concepts. On the other side, we see that very few proposals $(19,44 \%)$ have proposed a new DSL. There is therefore a clear preference of extending existing languages over defining new ones.

From our study we can conclude that current trends to include physical devices into business processes are based on the extension of the BPMN meta-model. BPMN extension mechanisms are conservative with its meta-model, allowing that current editors and engines can work with extended versions. However, only core concepts of its meta-model can be interpreted. In order to interpret new concepts additional effort to adapt editors and engines is required. To do that, however, a standardization effort should be performed in order to adopt a unique solution.

Another important aspect that has been observed in some of the analyzed studies is the application of the separation of concerns $(\mathrm{SoC})$ design principle. If $\mathrm{BP}$ models are extended or enriched with too much information we run the risk of making models no longer understandable. For this reason, specifying IoT devices separately from the BP model may also be a solution to keep BP models understandable for all the involved stakeholders.

Regarding RQ2, we have learned that IoT devices can be represented in the BP model either explicitly, i.e., by providing a specific construct to represent such element or implicitly, i.e., by means of another modeling element. When the representation is performed explicitly, the most natural way to represent such elements is by using or extending the participant or resource concept supported by BP modeling languages, which is graphically represented for example in BPMN as pools or lanes, but also with the definition of new modeling constructs. The analysis showed that 19,44\% of the studies opted for each of these solutions. However, other mechanisms to achieve the explicit representation is by reusing/extending existing concepts such as data objects. Finally, externalizing these elements from the BP model is also a solution designed by some of the studies $(13,89 \%)$. In this case, IoT devices are represented in detail in a separated model and linked afterwards to the BP model to reference them. Regarding the implicit representation, this is mainly achieved through the use of tasks and events, being this last element the most preferred by the analyzed studies $(22,22 \%)$. In this case, instead of representing the IoT device itself, it is represented in terms of its interaction with the BP model. To this end, while the use of tasks allows representing synchronous interactions with the device when it is required by the $\mathrm{BP}$, the use of events allows representing asynchronous interactions which are based on the occurrence of relevant situations of the device or environment.

Another very interesting finding relates to the abstraction level in which IoT devices are represented in a BP model. IoT scenarios operate in a very low abstraction level, being highly dependent on the device's technology. However, BP models should not be created based on the limitations imposed by such technology. Otherwise, changes in the underlying technology (which is constantly progressing) would require continuously revising and modifying the associated BP models. While Wehlitz2017 proposes to provide this abstraction by defining at the modeling level device types instead of instances to define technology independent models, other works have opted for the implementation of a middleware that makes the BPs independent of the device technology. This separation allows having BP model representations independent from the underlying technology and that can be reused in a different technological scenarios.

\section{STATE OF THE ART}

To the best of our knowledge, no SMS focused on the topic addressed in ours has been performed so far in the literature. However, during the primary studies search process we found some papers that although they pursue a different goal, share some similarities with this SMS. These papers are [55], [56], and [57] and focus their analysis on Mobile Cloud Computing (MCC), Wireless Sensor Network (WSN) applications, and Mobile Devices respectively.

The work developed by Chang et al. [55] is focused on the analysis and evaluation of a selection of BPMS for IoT (BPMS4IoT) frameworks from the MCC perspective. The analysis is organized based on the three different phases that conform the BPMS4IoT lifecycle (re-design, implementation/configuration, and execution and adjustment). Focused on the re-design analysis, which is the one related to the modeling stage addressed in this SMS, this paper analyzes seven works that are also considered in our SMS. Besides the small number of studies considered in the analysis regarding the modelling of IoT elements, all of them provide solutions based on the BPMN modeling language.

The work developed by Teixeira et al. [57] presents a SMS on how to model and automate code generation by the application of model-driven and business process approaches for WSN applications. This SMS discusses how WSN application requirements can be described in a BP representation. The major difference between this work and our SMS is the specific target of its analysis towards WSN-based IoT solutions. Teixera et al. limit their study to WSN applications and model-driven techniques that can be used to automatically generate code for the development of such applications.

Finally, the work developed by Dörndorfer2018 et al. [56] presents a study that identifies the impact that the use of a specific type of IoT device has on BPs and their lifecycle. Specifically, the focus is put on the context and how can be represented by the corresponding modeling languages. The 
major difference with our SMS is that this work puts the focus around context in BPs that are supported by mobile devices. To this end it explores the impact that context has on the different phases of BPs.

\section{CONCLUSIONS}

This work aims at providing a fundamental understanding of how to achieve the modeling of IoT-enhanced BPs. We performed a SMS that resulted in a total of 36 primary studies. These selected studies were analyzed according to two RQs which were focused on the specific aspects that deal with the integration of the IoT and the BPM at the modeling level. We identified the different strategies used to address the modeling of such BPs with to understand to which extent existing BP modeling languages could be used for such purpose and also how. Then, we looked more specifically at the mechanisms provided to introduce IoT devices into BP models.

Our future work will extend this SMS with additional research questions that analyze aspects such as the management of context in IoT-enhanced business processes, the validation performed by the different approaches, or the provided modelling tool support. In addition, it would be very interesting to study the different frameworks and architectures proposed in the literature to support a specific aspect found in many IoT scenarios, which is dealing with tons of data and events generated during BP execution.

\section{ACKNOWLEDGMENT}

This research has been funded by Internal Funds KU Leuven (Interne Fondsen KU Leuven) and the financial support of the Spanish State Research Agency under the project TIN2017-84094-R and co-financed with ERDF.

\section{REFERENCES}

[1] Ashton, K.: That 'internet of things' thing. RFID Journal (2009)

[2] Weske, M.: Business Process Management: Concepts, Languages, Architectures. Springer (2007)

[3] Janiesch, C., Koschmider, A., Mecella, M., Weber, B., Burattin, A., Ciccio, C.D., Gal, A., Kannengiesser, U., Mannhardt, F., Mendling, J., Oberweis, A., Reichert, M., Rinderle-Ma, S., Song, W., Su, J., Torres, V., Weidlich, M., Weske, M., Zhang, L.: The internet-of-things meets business process management: Mutual benefits and challenges. CoRR abs/1709.03628 (2017)

[4] Grefen, P., Ludwig, H., Tata, S., Dijkman, R., Baracaldo, N., Wilbik, A., d'Hondt, T.: Complex collaborative physical process management: A position on the trinity of bpm, iot and da. In: 19th IFIP/SOCOLNET Working Conference on Virtual Enterprises, Volume 534, 2018, Cardiff, UK, Sep 17-19, 2018. (2018)

[5] Wehlitz, R., Rößner, I., Franczyk, B.: Integrating smart devices as business process resources - concept and software prototype. In Braubach, L., Murillo, J.M., Kaviani, N., Lama, M., Burgueño, L., Moha, N., Oriol, M., eds.: Service-Oriented Computing - ICSOC 2017 Workshops - ASOCA, ISyCC, WESOACS, and Satellite Events, Málaga, Spain, November 13-16, 2017, Revised Selected Papers. Volume 10797 of Lecture Notes in Computer Science., Springer (2017) 252-257

[6] Tu, M., Lim, M.K., Yang, M.: Iot-based production logistics and supply chain system - part 1: Modeling iot-based manufacturing supply chain. Industrial Management and Data Systems 118 (2018) 65-95

[7] Schönig, S., Ackermann, L., Jablonski, S., Ermer, A.: An integrated architecture for iot-aware business process execution. In Gulden, J., Reinhartz-Berger, I., Schmidt, R., Guerreiro, S., Guédria, W., Bera, P., eds.: Enterprise, Business-Process and Information Systems Modeling 19th International Conference, BPMDS 2018, 23rd International Conference, EMMSAD 2018, Held at CAiSE 2018, Tallinn, Estonia, June 11-12, 2018, Proceedings. Volume 318 of Lecture Notes in Business Information Processing., Springer (2018) 19-34
[8] Dadam, P., Reichert, M.: The ADEPT project: a decade of research and development for robust and flexible process support. Comp Scie - R\&D 23 (2009) 81-97

[9] Chiu, H., Wang, M.: Extending event elements of business process model for internet of things. In: 15th IEEE International Conference on Computer and Information Technology, CIT 2015; 14th IEEE International Conference on Ubiquitous Computing and Communications, IUCC 2015; 13th IEEE International Conference on Dependable, Autonomic and Secure Computing, DASC 2015; 13th IEEE International Conference on Pervasive Intelligence and Computing, PICom 2015, Liverpool, United Kingdom, October 26-28, 2015. (2015) 783-788

[10] Meyer, S., Ruppen, A., Hilty, L.M.: The things of the internet of things in BPMN. In Persson, A., Stirna, J., eds.: Advanced Information Systems Engineering Workshops - CAiSE 2015 International Workshops, Stockholm, Sweden, June 8-9, 2015, Proceedings. Volume 215 of Lecture Notes in Business Information Processing., Springer (2015) 285-297

[11] Cheng, Y., Zhao, S., Cheng, B., Chen, X., Chen, J.: Modeling and deploying iot-aware business process applications in sensor networks. Sensors 19 (2019) 111

[12] Domingos, D., Martins, F.: Using bpmn to model internet of things behavior within business process. International Journal of Project Management 5 (2017) 39-51

[13] Suri, K., Gaaloul, W., Cuccuru, A.: Configurable iot-aware allocation in business processes. In Ferreira, J.E., Spanoudakis, G., Ma, Y., Zhang, L., eds.: Services Computing - SCC 2018 - 15th International Conference, Held as Part of the Services Conference Federation, SCF 2018, Seattle, WA, USA, June 25-30, 2018, Proceedings. Volume 10969 of Lecture Notes in Computer Science., Springer (2018) 119-136

[14] Gubbi, J., Buyya, R., Marusic, S., Palaniswami, M.: Internet of things (iot): A vision, architectural elements, and future directions. Future Generation Comp. Syst. 29 (2013) 1645-1660

[15] Dorsemaine, B., Gaulier, J., Wary, J., Kheir, N., Urien, P.: Internet of things: A definition amp;amp; taxonomy. In: 2015 9th International Conference on Next Generation Mobile Applications, Services and Technologies. (2015) 72-77

[16] Rayes, A., Salam, S. In: Internet of Things (IoT) Overview. Springer International Publishing, Cham (2017) 1-34

[17] Ray, P.: A survey on internet of things architectures. Journal of King Saud University - Computer and Information Sciences 30 (2018) 291 319

[18] Kitchenham, B., Charters, S.: Guidelines for performing systematic literature reviews in software engineering. Technical report, Technical Report EBSE-2007-01. School of Computer Science and Mathematics, Keele University. (2007)

[19] Wohlin, C.: Guidelines for snowballing in systematic literature studies and a replication in software engineering. In Shepperd, M.J., Hall, T., Myrtveit, I., eds.: 18th International Conference on Evaluation and Assessment in Software Engineering, EASE '14, London, England, United Kingdom, May 13-14, 2014, ACM (2014) 38:1-38:10

[20] Zhang, H., Babar, M.A., Tell, P.: Identifying relevant studies in software engineering. Information \& Software Technology 53 (2011) 625-637

[21] Al-alshuhai, A., Siewe, F.: An extension of UML activity diagram to model the behaviour of context-aware systems. In Wu, Y., Min, G., Georgalas, N., Hu, J., Atzori, L., Jin, X., Jarvis, S.A., Liu, L.C., Calvo, R.A., eds.: 15th IEEE International Conference on Computer and Information Technology, CIT 2015; 14th IEEE International Conference on Ubiquitous Computing and Communications, IUCC 2015; 13th IEEE International Conference on Dependable, Autonomic and Secure Computing, DASC 2015; 13th IEEE International Conference on Pervasive Intelligence and Computing, PICom 2015, Liverpool, United Kingdom, October 26-28, 2015, IEEE (2015) 431-437

[22] Albreshne, A., Pasquier, J.: A domain specific language for high-level process control programming in smart buildings. In: The 6th International Conference on Emerging Ubiquitous Systems and Pervasive Networks (EUSPN 2015)/ The 5th International Conference on Current and Future Trends of Information and Communication Technologies in Healthcare (ICTH-2015)/ Affiliated Workshops, September 27-30, 2015, Berlin, Germany. Volume 63 of Procedia Computer Science., Elsevier (2015) 65-73

[23] Appel, S., Kleber, P., Frischbier, S., Freudenreich, T., Buchmann, A.: Modeling and execution of event stream processing in business processes. Information Systems 46 (2014) 140 - 156

[24] Baresi, L., Meroni, G., Plebani, P.: A gsm-based approach for monitoring cross-organization business processes using smart objects. In Reichert, 
M., Reijers, H.A., eds.: Business Process Management Workshops BPM 2015, 13th International Workshops, Innsbruck, Austria, August 31 - September 3, 2015, Revised Papers. Volume 256 of Lecture Notes in Business Information Processing., Springer (2015) 389-400

[25] Bocciarelli, P., D’Ambrogio, A., Giglio, A., Paglia, E.: A BPMN extension for modeling cyber-physical-production-systems in the context of industry 4.0. In Fortino, G., Zhou, M., Lukszo, Z., Vasilakos, A.V., Basile, F., Palau, C.E., Liotta, A., Fanti, M.P., Guerrieri, A., Vinci, A., eds.: 14th IEEE International Conference on Networking, Sensing and Control, ICNSC 2017, Calabria, Italy, May 16-18, 2017, IEEE (2017) 599-604

[26] Breitenbücher, U., Hirmer, P., Képes, K., Kopp, O., Leymann, F., Wieland, M.: A situation-aware workflow modelling extension. In Proceedings of the 17th International Conference on Information Integration and Web-based Applications \& Services, iiWAS 2015, Brussels, Belgium, December 11-13, 2015. (2015) 64:1-64:7

[27] Bucchiarone, A., Lluch-Lafuente, A., Marconi, A., Pistore, M.: A formalisation of adaptable pervasive flows. In Laneve, C., Su, J., eds.: Web Services and Formal Methods, 6th International Workshop, WSFM 2009, Bologna, Italy, September 4-5, 2009, Revised Selected Papers. Volume 6194 of Lecture Notes in Computer Science., Springer (2009) $61-75$

[28] Caracaş, A., Kramp, T.: On the expressiveness of bpmn for modeling wireless sensor networks applications. In Dijkman, R., Hofstetter, J., Koehler, J., eds.: Business Process Model and Notation, Berlin, Heidelberg, Springer Berlin Heidelberg (2011) 16-30

[29] Dar, K., Taherkordi, A., Baraki, H., Eliassen, F., Geihs, K.: A resource oriented integration architecture for the internet of things: A business process perspective. Pervasive and Mobile Computing 20 (2015) 145159

[30] Domingos, D., Martins, F., Cândido, C., Martinho, R.: Internet of things aware ws-bpel business processes context variables and expected exceptions. J. UCS 20 (2014) 1109-1129

[31] Dörndorfer, J., Seel, C.: A framework to model and implement mobile context-aware business applications. In Schaefer, I., Karagiannis, D., Vogelsang, A., Méndez, D., Seidl, C., eds.: Modellierung 2018, $21 .-$ 23. Februar 2018, Braunschweig, Germany. Volume P-280 of LNI., Gesellschaft für Informatik e.V. (2018) 23-38

[32] Friedow, C., Völker, M., Hewelt, M.: Integrating iot devices into business processes. In Matulevicius, R., Dijkman, R.M., eds.: Advanced Information Systems Engineering Workshops - CAiSE 2018 International Workshops, Tallinn, Estonia, June 11-15, 2018, Proceedings. Volume 316 of Lecture Notes in Business Information Processing., Springer (2018) 265-277

[33] Gao, F., Zaremba, M., Bhiri, S., Derguerch, W.: Extending bpmn 2.0 with sensor and smart device business functions. In: 2011 IEEE 20th International Workshops on Enabling Technologies: Infrastructure for Collaborative Enterprises. (2011) 297-302

[34] Graja, I., Kallel, S., Guermouche, N., Kacem, A.H.: Bpmn4cps: A bpmn extension for modeling cyber-physical systems. In: 2016 IEEE 25th International Conference on Enabling Technologies: Infrastructure for Collaborative Enterprises (WETICE). (2016) 152-157

[35] Kefalakis, N., Soldatos, J., Konstantinou, N., Prasad, N.R.: APDL: A reference XML schema for process-centered definition of RFID solutions. Journal of Systems and Software 84 (2011) 1244-1259

[36] Kim, S.D., Lee, J.Y., Kim, D.Y., Park, C.W., La, H.J.: Modeling bpelbased collaborations with heterogeneous iot devices. In: 2014 IEEE 12th International Conference on Dependable, Autonomic and Secure Computing. (2014) 289-294

[37] Kim, M., Ahn, H., Kim, K.P.: Process-aware internet of things: A conceptual extension of the internet of things framework and architecture. TIIS 10 (2016) 4008-4022

[38] Lee, W., Ma, S.: Process modeling and analysis of service-oriented architecture-based wireless sensor network applications using multipledomain matrix. IJDSN 12 (2016)

[39] Maamar, Z., Sellami, M., Faci, N., Ugljanin, E., Sheng, Q.Z.: Storytelling integration of the internet of things into business processes. In Weske, M., Montali, M., Weber, I., vom Brocke, J., eds.: Business Process Management Forum - BPM Forum 2018, Sydney, NSW, Australia, September 9-14, 2018, Proceedings. Volume 329 of Lecture Notes in Business Information Processing., Springer (2018) 127-142

[40] Mandal, S., Hewelt, M., Weske, M.: A framework for integrating real-world events and business processes in an iot environment. In Panetto, H., Debruyne, C., Gaaloul, W., Papazoglou, M.P., Paschke,
A., Ardagna, C.A., Meersman, R., eds.: On the Move to Meaningful Internet Systems. OTM 2017 Conferences - Confederated International Conferences: CoopIS, C\&TC, and ODBASE 2017, Rhodes, Greece, October 23-27, 2017, Proceedings, Part I. Volume 10573 of Lecture Notes in Computer Science., Springer (2017) 194-212

[41] Meyer, S., Ruppen, A., Magerkurth, C.: Internet of things-aware process modeling: Integrating iot devices as business process resources. In Salinesi, C., Norrie, M.C., Pastor, O., eds.: Advanced Information Systems Engineering - 25th International Conference, CAiSE 2013, Valencia, Spain, June 17-21, 2013. Proceedings. Volume 7908 of Lecture Notes in Computer Science., Springer (2013) 84-98

[42] Mottola, L., Picco, G.P., Opperman, F.J., Eriksson, J., Finne, N., Fuchs, H., Gaglione, A., Karnouskos, S., Montero, P., Oertel, N., Römer, K., Spiess, P., Tranquillini, S., Voigt, T.: makesense: Simplifying the integration of wireless sensor networks into business processes. IEEE Transactions on Software Engineering (2018) 1-1

[43] Petrasch, R., Hentschke, R.: Process modeling for industry 4.0 applications: Towards an industry 4.0 process modeling language and method. In: 2016 13th International Joint Conference on Computer Science and Software Engineering (JCSSE). (2016) 1-5

[44] Sasirekha, S., Swamynathan, S.: Collaboration of iot devices using semantically enabled resource oriented middleware. In: Proceedings of the Third International Symposium on Computer Vision and the Internet. VisionNet'16, New York, NY, USA, ACM (2016) 98-105

[45] Seiger, R., Keller, C., Niebling, F., Schlegel, T.: Modelling complex and flexible processes for smart cyber-physical environments. Journal of Computational Science 10 (2015) $137-148$

[46] Serral, E., Smedt, J.D., Snoeck, M., Vanthienen, J.: Context-adaptive petri nets: Supporting adaptation for the execution context. Expert Systems with Applications 42 (2015) 9307 - 9317

[47] Sperner, K., Meyer, S., Magerkurth, C.: Introducing entity-based concepts to business process modeling. In Dijkman, R., Hofstetter, J., Koehler, J., eds.: Business Process Model and Notation, Berlin, Heidelberg, Springer Berlin Heidelberg (2011) 166-171

[48] Suri, K., Gaaloul, W., Cuccuru, A., Gerard, S.: Semantic framework for internet of things-aware business process development. In: 2017 IEEE 26th International Conference on Enabling Technologies: Infrastructure for Collaborative Enterprises (WETICE). (2017) 214-219

[49] Wang, P., Zhang, H.L.B.: A context-aware workflow framework and modeling language. 175 (2014) 198-206

[50] Yousfi, A., Batoulis, K., Weske, M.: Achieving business process improvement via ubiquitous decision-aware business processes. ACM Trans. Internet Techn. 19 (2019) 14:1-14:19

[51] Workflow Management Coalition Workflow Standard: Process definition interface - $\mathrm{xml}$ process definition language v1.0. Standard WFMC-TC1025, The Workflow Management Coalition (2002)

[52] Bostan, B., Marsh, T.: Fundamentals of interactive storytelling. Academic Journal of Information Technology 3 (2012)

[53] Dimitrov, M., Simov, A., Stein, S., Konstantinov, M.: A BPMO based semantic business process modelling environment. In Hepp, M., Hinkelmann, K., Karagiannis, D., Klein, R., Stojanovic, N., eds.: Proceedings of the Workshop on Semantic Business Process and Product Lifecycle Management SBPM 2007, held in conjunction with the 3rd European Semantic Web Conference (ESWC 2007), Innsbruck, Austria, June 7, 2007. Volume 251 of CEUR Workshop Proceedings., CEURWS.org (2007)

[54] Bermúdez-Edo, M., Elsaleh, T., Barnaghi, P.M., Taylor, K.: Iot-lite: a lightweight semantic model for the internet of things and its use with dynamic semantics. Personal and Ubiquitous Computing 21 (2017) 475487

[55] Chang, C., Srirama, S.N., Buyya, R.: Mobile cloud business process management system for the internet of things: A survey. ACM Comput. Surv. 49 (2016) 70:1-70:42

[56] Dörndorfer, J., Seel, C.: Research agenda for mobile context sensitive business processes. AKWI 6 (2017) 20-29

[57] Teixeira, S., Agrizzi, B.A., Filho, J.G.P., Rossetto, S., de Lima Baldam, R.: Modeling and automatic code generation for wireless sensor network applications using model-driven or business process approaches: A systematic mapping study. Journal of Systems and Software 132 (2017) $50-71$ 